\title{
Periodic minimizers in 1D local mean field theory
}

\author{
Alessandro Giuliani* \\ Department of Physics, Princeton University, Princeton 08544 NJ, USA.
}

Joel L. Lebowitz

Departments of Mathematics and Physics, Rutgers University, Piscataway, NJ 08854 USA.

Elliott H. Lieb

Departments of Mathematics and Physics, Princeton University, Princeton, NJ 08544 USA.

(Dated: December 13, 2007)

\begin{abstract}
Using reflection positivity techniques we prove the existence of minimizers for a class of mesoscopic free-energies representing 1D systems with competing interactions. All minimizers are either periodic, with zero average, or of constant sign. If the local term in the free energy satisfies a convexity condition, then all minimizers are either periodic or constant. Examples of both phenomena are given. This extends our previous work where such results were proved for the ground states of lattice systems with ferromagnetic nearest neighbor interactions and dipolar type antiferromagnetic long range interactions.
\end{abstract}

\section{INTRODUCTION}

We consider the nature of the minimizers for a class of $1 \mathrm{D}$ free-energy functionals that model the continuum limit of microscopic systems with competing interactions on different length scales. An example is a spin system on a lattice with a nearest neighbor ferromagnetic interaction and a long range antiferromagnetic power law type interaction. In [17] we considered

*Present Address Dipartimento di Matematica di Roma Tre, Largo S. Leonardo Murialdo 1, 00146 Roma, Italy.

(C) 2007 by the authors. This paper may be reproduced, in its entirety, for non-commercial purposes. 
the ground states of such systems in one-dimension and in [18] we also investigated higher dimensional models with dipolar type interactions. In both cases we obtained periodic ground states whose period (which could be infinite) depended on both the strength of the short range interaction and the nature of the long range interaction. The technique used in those papers, reflection positivity, could not be extended to positive temperatures, for which only approximate methods and computer simulations are available now $[1,19,24,31]$. It turns out, however, that these reflection positivity methods are directly applicable to the Ginzburg-Landau type freeenergy functionals used to describe the continuum versions of such microscopic systems [26, 28]. These include finite temperature effects, at least at a mean field level, via an inclusion of a local entropy term in the effective continuum description of the system.

These free-energies functionals have been used extensively in both the physical and mathematical literatures as models for a great variety of systems, including micromagnets $[7,10,14]$, diblock copolymers [20, 23, 27], anisotropic electron gases [29, 30], polyelectrolytes [5], chargedensity waves in layered transition metals [25] and superconducting films [12]. Many of these systems are characterized by low temperature phases displaying spontaneous formation of periodic mesoscopic patterns, such as stripes or bubbles. It is, therefore, important to show that these free energy functionals have periodic minimizers. This has been proved rigorously in some cases $[2,9]$ and argued for heuristically in others $[6,7,12,14,16,20,23,26,29,30]$.

In this paper we use reflection positivity methods to prove the periodicity of minimizers for a certain class of such $1 \mathrm{D}$ free-energy functionals. These include cases that were not treated before, e.g., those with long-range power law type interactions. As noted before, reflection positivity methods have been succesfully applied to find periodic ground states for a class of microscopic 1D and 2D lattice spin models but has not, as far as we know, been used before for continuum systems. We begin in Section 2 by presenting the class of models under consideration and our results. These are proved in Section 3. In Section 4 we give an example of the transition from a state of finite periodicity to a uniform (infinite periodicity) state, as a parameter is varied. We discuss the connection with related work in Section 5. 


\section{FORMULATION OF MODEL AND STATEMENT OF RESULTS}

The formal infinite volume free energy functional to be minimized, in a sense to be made precise below, is

$\mathcal{E}(\phi)=\int_{\mathbb{R}} d x\left[\left(\phi^{\prime}(x)\right)^{2}+F(\phi)\right]+\int_{\mathbb{R}} d x \int_{\mathbb{R}} d y \phi(x) v(x-y) \phi(y), \quad v(x)=\lambda \int_{0}^{\infty} \nu(d \alpha) e^{-|x| \alpha}$

with $\nu(d \alpha)$ a probability measure such that $\lambda \int_{0}^{\infty} \nu(d \alpha) \alpha^{-1}=\int_{0}^{\infty} v(x) d x<+\infty, \lambda$ a positive constant. We shall also assume that $F(t)$ is an even function of its argument, and that $F(t) \geq 0$, with $F(t)>0$ for $|t|<1$ and $F(t)=0$ for $|t|=1$. Note that we do not need either that $F$ is continuous or that it goes to $+\infty$ as $|t| \rightarrow \infty$. Some examples to keep in mind are $F(t)=\left(t^{2}-1\right)^{2}$ or $F(t)=(|t|-1)^{2}$ or $F(t)=a(t)-a(1)$, where, defining $\alpha=\tanh \beta$ :

$$
a(t)= \begin{cases}-t^{2}+(\alpha \beta)^{-1}[(1+\alpha t) \log (1+\alpha t)+(1-\alpha t) \log (1-\alpha t)], & \text { if }|t|<\alpha^{-1} \\ +\infty, & \text { if }|t| \geq \alpha^{-1}\end{cases}
$$

The gradient term in (2.1) represents the cost of a transition between two phases, while the term $F$ represents the local free energy density for a homogeneous system. We have chosen $F(t)$ to have two symmetric minima corresponding to the case of a ferromagnetic Ising spin system (and its analogues) below $T_{c}$. (Since we are not concerned here explicitly with the dependence on temperature we have used the scaling parameter $\alpha$ to set the value of the mean field spontaneous magnetization in $a(t)$, at $T<T_{c}$, equal to unity.) The third term on the right represents the long range antiferromagnetic type interaction. Note that $v$, which can include terms decaying as a power law, is reflection-positive, see [13], summable and completely monotone, i.e., $(-1)^{n} v^{(n)}(x) \geq 0$ and $\searrow 0$ as $x \rightarrow \infty$. The minimum value of this interaction term occurs when $\phi=0$. It competes, therefore, with the first two terms, which are minimized when $\phi(x)=1$ or $\phi(x)=-1$ for all $x$. (Note, however, that in the absence of the gradient square term the minimizer would be an infinitely rapidly oscillating $\phi(x)$, between -1 and +1 ; see discussion of Kac potential in Section 5.)

To state our results, let us first recall some standard notation. $H^{1}(\mathbb{R})$ is the space of functions whose first distributional derivative is in $L^{2}(\mathbb{R})$ and which $\rightarrow 0$ as $|x| \rightarrow \infty$. $H_{\text {loc }}^{1}(\mathbb{R})$ is the space of functions whose derivatives are in $L^{2}([a, b])$ for all intervals $-\infty<a<b<+\infty$ and $H_{0}^{1}([a, b])$ are $H^{1}$ functions that vanish on the endpoints $a, b$. In one dimension, $H^{1}$ 
functions are equivalent to Hölder continuous functions, with Hölder exponent 1/2. (See [21]). We next define the notions of infinite volume specific ground state free energy and of infinite volume ground state.

Definition 1. Given a finite interval $[a, b]$ on the real line, let $\mathcal{E}_{a, b}^{\mathrm{F}}: H^{1}([a, b]) \rightarrow \mathbb{R}^{+}$be the finite volume functional with free boundary conditions, defined as

$$
\mathcal{E}_{a, b}^{\mathrm{F}}(\phi)=\int_{a}^{b} d x\left[\left(\phi^{\prime}(x)\right)^{2}+F(\phi)\right]+\int_{a}^{b} d x \int_{a}^{b} d y \phi(x) v(x-y) \phi(y) .
$$

Moreover, let $\mathcal{E}_{a, b}^{\mathrm{D}}$ be the restriction of $\mathcal{E}_{a, b}^{\mathrm{F}}$ to $H_{0}^{1}([a, b])$, that is the finite volume functional with Dirichlet boundary conditions $\phi(a)=\phi(b)=0$. Let

$$
\begin{aligned}
& E_{L}^{\mathrm{F}} \equiv \inf _{\phi \in H^{1}([0, L])} \mathcal{E}_{0, L}^{\mathrm{F}}(\phi) \\
& E_{L}^{\mathrm{D}} \equiv \inf _{\phi \in H_{0}^{1}([0, L])} \mathcal{E}_{0, L}^{\mathrm{D}}(\phi) .
\end{aligned}
$$

Then we define the infinite volume specific ground state free energy $e_{0}$ corresponding to the formal energy functional $\mathcal{E}(\phi)$ to be

$$
e_{0}=\lim _{L \rightarrow \infty} E_{L}^{\mathrm{F}} / L=\lim _{L \rightarrow \infty} E_{L}^{\mathrm{D}} / L
$$

whenever the limits on the r.h.s. exist and are equal.

Definition 2. Given $\phi \in H_{\text {loc }}^{1}(\mathbb{R})$, then, for any interval $[a, b]$ on the real line, we define:

$$
\begin{aligned}
\mathcal{E}_{a, b}(\phi)=\int_{a}^{b} d x\left[\left(\phi^{\prime}(x)\right)^{2}+F(\phi)\right] & +\int_{a}^{b} d x \int_{a}^{b} d y \phi(x) v(x-y) \phi(y)+ \\
& +2 \int_{a}^{b} d x \int_{\mathbb{R} \backslash[a, b]} d y \phi(x) v(x-y) \phi(y) .
\end{aligned}
$$

We say that $\phi_{0} \in H_{\text {loc }}^{1}(\mathbb{R})$ is an infinite volume ground state of $\mathcal{E}(\phi)$ if $\mathcal{E}_{a, b}\left(\phi_{0}\right) \leq \mathcal{E}_{a, b}(\psi)$, for all intervals $[a, b]$ and all functions $\psi$ coinciding with $\phi_{0}$ on $\mathbb{R} \backslash[a, b]$.

In the following we shall exploit the reflection-positivity of $v$ in order to show existence of periodic minimizers for the functional (2.1). We need to introduce some more definitions and notation.

Definition 3. Let $M, N \in \mathbb{Z}^{+} \cup\{+\infty\}$ be such that $M+N \geq 1$. Let $\mathcal{F}=\left\{f_{-M+1}, \ldots, f_{0}, f_{1}, \ldots, f_{N}\right\}$ be a sequence of functions such that $f_{i} \in H_{0}^{1}\left(\left[0, T_{i}\right]\right)$ 
and $T_{i}>0$, with $-M<i \leq N$. Let $x_{-M}=-\sum_{j=-M+1}^{0} T_{j}$ and $x_{i}=x_{-M}+\sum_{j=-M+1}^{i} T_{j}$, for all $-M<i \leq N$ (if $M=0$ it is understood that $\left.x_{0}=0\right)$. Then we define $\varphi[\mathcal{F}] \in H_{0}^{1}\left(\left[x_{-M}, x_{N}\right]\right)$ to be the function obtained by juxtaposing the functions $f_{i}$ on the real line, in such a way that, if $x_{i-1} \leq x \leq x_{i}$, then $\varphi[\mathcal{F}](x)=f_{i}\left(x-x_{i-1}\right)$, for all $i=-M+1, \ldots, N$.

In order to visualize the meaning of Definition 3, we plot, in Figure 1, a function $\varphi[\mathcal{F}](x)$ corresponding to $M=N=2$.

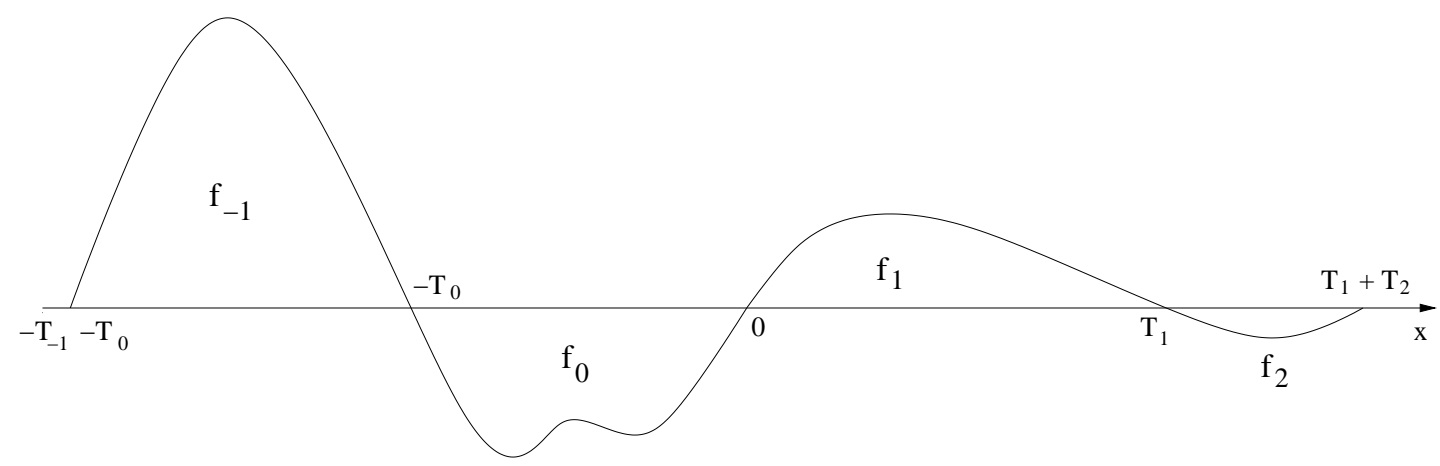

FIG. 1: A possible function $\varphi[\mathcal{F}]$ before reflection.

Definition 4. (i) Given $T>0$ and $f \in H_{0}^{1}([0, T])$, we define $\theta f \in H_{0}^{1}([0, T])$ to be the reflection of $f$, namely $\theta f(x)=-f(T-x)$, for all $x \in[0, T]$.

(ii) If $f \in H_{0}^{1}([0, T])$, we define $\varphi[f]=\varphi\left[\mathcal{F}_{\infty}(f)\right] \in H_{\text {loc }}^{1}(\mathbb{R})$, where $\mathcal{F}_{\infty}(f)=\left\{\ldots, f_{0}, f_{1}, \ldots\right\}$ is the infinite sequence with $f_{n}=\theta^{n-1} f$.

(iii) Given a sequence $\mathcal{F}=\left\{f_{-M+1}, \ldots, f_{N}\right\}$ as in Def.3, we define $\mathcal{F}_{-}=\left\{f_{-M+1}, \ldots, f_{0}\right\}$ and $\mathcal{F}_{+}=\left\{f_{1}, \ldots, f_{N}\right\}$ (if $M=0$ or $N=0$, it is understood that $\mathcal{F}_{-}$or, respectively, $\mathcal{F}_{+}$is empty) and we write $\mathcal{F}=\left(\mathcal{F}_{-}, \mathcal{F}_{+}\right)$.

(iv) The reflections of $\mathcal{F}_{-}$and $\mathcal{F}_{+}$are defined to be: $\theta \mathcal{F}_{-}=\left\{\theta f_{0}, \ldots, \theta f_{-M+1}\right\}$ and $\theta \mathcal{F}_{+}=\left\{\theta f_{N}, \ldots, \theta f_{1}\right\}$. See Figure 2.

We are now ready to state our main results.

Theorem 1. (Specific ground state free energy). For any $T>0$, let $\mathcal{C}_{T}=\{f \in$ 

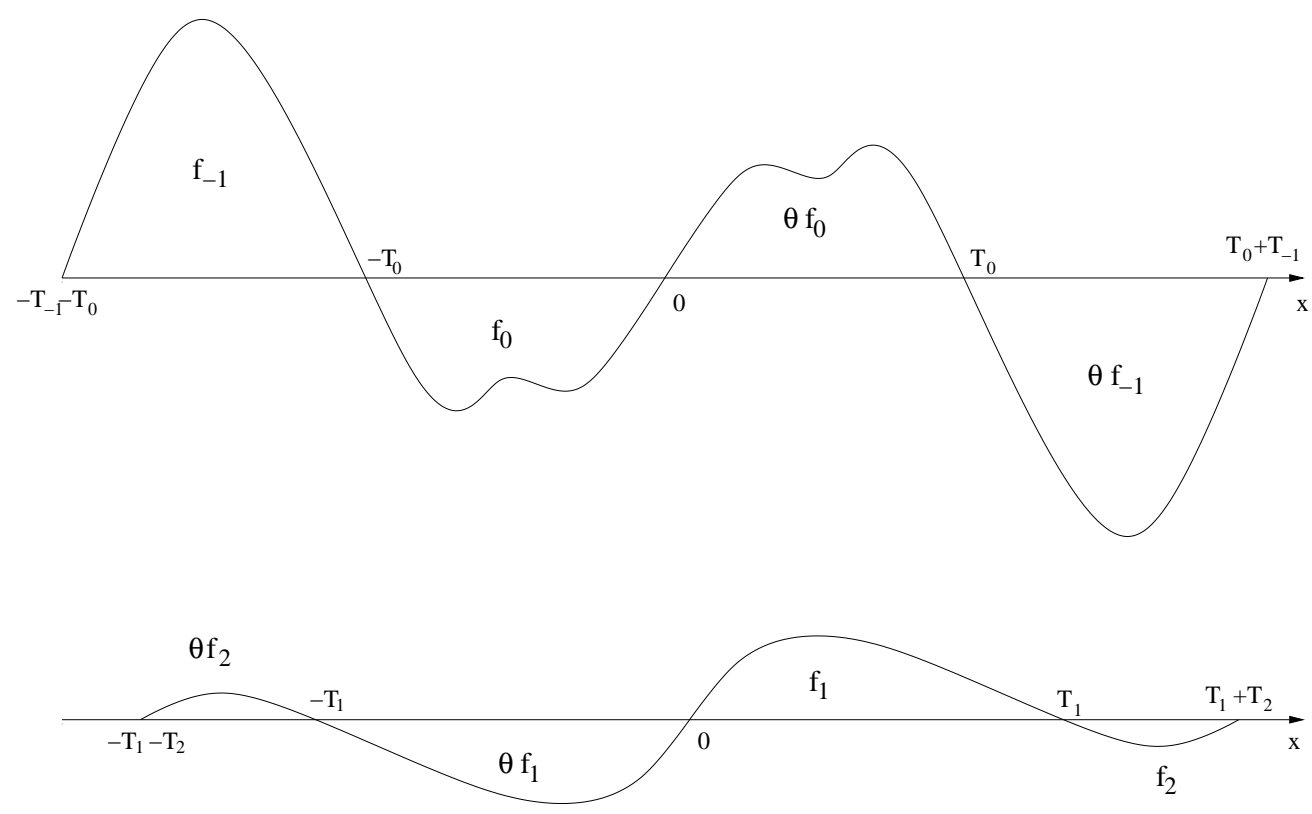

FIG. 2: The two reflected configurations $\varphi\left[\mathcal{F}_{1}\right]$ and $\varphi\left[\mathcal{F}_{2}\right]$ obtained from the function $\varphi[\mathcal{F}]$ in Fig.1 after reflection around 0 .

$\left.H_{0}^{1}([0, T]): f \geq 0\right\}$. The limits in (2.5) exist, are equal and are given by

$$
e_{0}=\inf _{T} e_{T}, \quad e_{T} \equiv \inf _{f \in \mathcal{C}_{T}} e_{\infty}(f)
$$

where,

$$
e_{\infty}(f)=\lim _{L \rightarrow \infty} \frac{\mathcal{E}_{-L, L}^{\mathrm{F}}(\varphi[f])}{2 L}
$$

(Note that the limit in the r.h.s. of (2.8) exists, because $\varphi[f]$ is periodic and $v$ is summable.)

Moreover $e_{T}$ is a continuous function of $T$ and $\lim _{T \rightarrow \infty} e_{T}$ exists and equals $e_{0}$. It is given by

$$
\lim _{T \rightarrow \infty} e_{T}=\inf _{f \in \mathcal{C}_{\infty}} \liminf _{L \rightarrow \infty} \frac{\mathcal{E}_{-L, L}^{\mathrm{F}}(f)}{2 L}
$$

where $\mathcal{C}_{\infty}=\left\{f \in H_{\text {loc }}^{1}(\mathbb{R}): 0 \leq f \leq 1\right\}$

There is a function, $\phi_{T}$ that is a minimizer for (2.7) and satisfies $\left|\phi_{T}\right| \leq 1$. If $F(t)$ is differentiable for $t>0$, then $\phi_{T}$ is twice differentiable and it satisfies the Euler-Lagrange equation

$$
\phi_{T}^{\prime \prime}(x)=\frac{1}{2} F^{\prime}\left(\phi_{T}(x)\right)+\int_{-\infty}^{+\infty} d y v(x-y)\left(\varphi\left[\phi_{T}\right]\right)(y)
$$

If $F(t)$ is convex for $t>0$, then $\phi_{T}$ is unique and the inf on the r.h.s. of (2.9) is a minimum, 
with a constant function as a minimizer.

Corollary 1. (Infinite volume ground states). (i) If there exists $T_{0}$ such that $e_{0}=e_{T_{0}}=e_{\infty}\left(\phi_{T_{0}}\right)$, then $\varphi\left[\phi_{T_{0}}\right]$ is an infinite volume ground state of $\mathcal{E}(\phi)$.

(ii) If $e_{0}=\lim _{T \rightarrow \infty} e_{T}$ and $F(t)$ is convex for $t \geq 0$, the constant function $\phi \equiv t_{0}$, with $t_{0}>0$ the point at which $F(t)+t^{2} \int_{-\infty}^{+\infty} v(x) d x$ achieves its minimum for $t>0$, is an infinite volume ground state of $\mathcal{E}(\phi)$. (Of course so is $\phi \equiv-t_{0}$.)

Remark. Theorem 1 and its Corollary may be informally stated by saying that all the minimizers of $\mathcal{E}(\phi)$ are either simply periodic, of finite period $T$, with zero average, or of constant sign (and are constant if $F$ is convex on $\mathbb{R}^{+}$). By "simply periodic" we mean that within a period the minimizer has only one positive and one negative region, with the negative part obtained by a reflection from the positive part.

\section{PROOF OF THE MAIN RESULTS}

As proved in [13], reflection-positivity of the long range potential $v$ implies the following basic estimate.

Lemma 1. Given a finite sequence of functions $\mathcal{F}=\left\{f_{-M+1} \ldots, f_{0}, f_{1}, \ldots, f_{N}\right\}=\left(\mathcal{F}_{-}, \mathcal{F}_{+}\right)$, as in Def.3 and 4, we have:

$$
\begin{gathered}
\mathcal{E}_{x_{-M}, x_{N}}^{\mathrm{D}}(\varphi[\mathcal{F}]) \geq \frac{1}{2} \mathcal{E}_{-x_{N}, x_{N}}^{\mathrm{D}}\left(\varphi\left[\mathcal{F}_{1}\right]\right)+\frac{1}{2} \mathcal{E}_{x_{-M},-x_{-M}}^{\mathrm{D}}\left(\varphi\left[\mathcal{F}_{2}\right]\right) \\
\text { where } \mathcal{F}_{1}=\left(\theta \mathcal{F}_{+}, \mathcal{F}_{+}\right)=\left\{\theta f_{N}, \ldots, \theta f_{1}, f_{1}, \ldots, f_{N}\right\} \text { and } \mathcal{F}_{2}=\left(\mathcal{F}_{-}, \theta \mathcal{F}_{-}\right)= \\
\left\{f_{-M+1}, \ldots, f_{0}, \theta f_{0}, \ldots, \theta f_{-M+1}\right\} .
\end{gathered}
$$

In terms of the function $\varphi[\mathcal{F}]$ in Figure 1, the statement of the Lemma is that the energy of this function is larger than the average of the energies of the two reflected configurations in Figure 2 .

The key technical ingredient in the proof of Theorem 1 is the chessboard estimate, which 
is obtained from Lemma 1 by repeatedly reflecting around different nodes of the function. A chessboard estimate in the presence of periodic boundary conditions has appeared many times before in the literature, see for instance [13]. Here, however, we will need a generalization of it to the case of Dirichlet boundary conditions, and we proceed as proposed in the Appendix of [18].

Chessboard estimate with Dirichlet boundary conditions. Given a finite sequence of functions $\mathcal{F}=\left\{f_{1}, \ldots, f_{N}\right\}, N \geq 1$, as in Definition 3, with $f_{i} \in H_{0}^{1}\left(\left[0, T_{i}\right]\right)$, we have:

$$
\mathcal{E}_{0, x_{N}}^{\mathrm{D}}(\varphi[\mathcal{F}]) \geq \sum_{i=1}^{N} T_{i} e_{\infty}\left(f_{i}\right) .
$$

Proof of (3.12). We proceed by induction.

(i) If $N=1$, let us first compare the energy of $f_{1}$ with that of $\left\{f_{1}, \pm \theta f_{1}\right\}$. Using the fact that $F(t)$ is even, the energy of $\left\{f_{1}, \pm \theta f_{1}\right\}$ can be rewritten as:

$$
\begin{aligned}
\mathcal{E}_{0,2 x_{1}}^{\mathrm{D}}\left(\varphi\left[\left\{f_{1}, \pm \theta f_{1}\right\}\right]\right) & =2 \mathcal{E}_{0, x_{1}}^{\mathrm{D}}\left(f_{1}\right) \pm 2 \int_{0}^{x_{1}} d x \int_{x_{1}}^{2 x_{1}} f_{1}(x) v(x-y) \theta f_{1}\left(y-x_{1}\right) \equiv \\
& \equiv 2 \mathcal{E}_{0, x_{1}}^{\mathrm{D}}\left(f_{1}\right)+E_{\text {int }}\left(f_{1} ; \pm \theta f_{1}\right) .
\end{aligned}
$$

At least one of the two interaction energies $E_{\text {int }}\left(f_{1} ; \theta f_{1}\right)$ or $E_{\text {int }}\left(f_{1} ;-\theta f_{1}\right)$ is $\leq 0$, simply because $E_{\text {int }}\left(f_{1} ;-\theta f_{1}\right)=-E_{\text {int }}\left(f_{1} ; \theta f_{1}\right)$. By reflection positivity, i.e., by Lemma 1 , we have in fact that $\mathcal{E}_{0,2 x_{1}}^{\mathrm{D}}\left(\varphi\left[\left\{f_{1}, \pm \theta f_{1}\right\}\right]\right) \geq \mathcal{E}_{0,2 x_{1}}^{\mathrm{D}}\left(\varphi\left[\left\{f_{1}, \theta f_{1}\right\}\right]\right)$, therefore $E_{\text {int }}\left(f_{1} ; \theta f_{1}\right) \leq 0$. Using $(3.13)$ we find:

$$
\mathcal{E}_{0, x_{1}}^{\mathrm{D}}\left(f_{1}\right) \geq \frac{1}{2} \mathcal{E}_{0,2 x_{1}}^{\mathrm{D}}\left(\varphi\left[\left\{f_{1}, \theta f_{1}\right\}\right]\right) .
$$

Iterating the same argument, we find:

$$
\mathcal{E}_{0, x_{1}}^{\mathrm{D}}\left(f_{1}\right) \geq \frac{\mathcal{E}_{0,2^{m} x_{1}}^{\mathrm{D}}\left(\varphi\left[f_{1}^{\otimes 2^{m}}\right]\right)}{2^{m}},
$$

where, by definition,

$$
f_{1}^{\otimes 2^{m}}=\{\overbrace{f_{1}, \theta f_{1}, \ldots, f_{1}, \theta f_{1}}^{2^{m}}\} .
$$

Taking the limit $m \rightarrow \infty$ in (3.15) we get the desired estimate:

$$
\mathcal{E}_{0, x_{1}}^{\mathrm{D}}\left(f_{1}\right) \geq T_{1} e_{\infty}\left(f_{1}\right)
$$

(ii) Let us now assume by induction that the bound is valid for all $1 \leq N \leq n-1, n \geq 2$, and let us prove it for $N=n$. There are two cases. 
(a) $n=2 p$ for some $p \geq 1$. If we reflect once, by Lemma 1 we have:

$$
\begin{aligned}
\mathcal{E}_{0, x_{2 p}}^{\mathrm{D}}\left(\varphi\left[\left\{f_{1}, \ldots, f_{2 p}\right\}\right]\right) & \geq \frac{1}{2} \mathcal{E}_{0,2\left(x_{2 p}-x_{p}\right)}^{\mathrm{D}}\left(\varphi\left[\left\{\theta f_{2 p}, \ldots, \theta f_{p+2},\left(\theta f_{p+1}\right)^{\otimes 2}, f_{p+2}, \ldots f_{2 p}\right\}\right]\right)+ \\
& +\frac{1}{2} \mathcal{E}_{0,2 x_{p}}^{\mathrm{D}}\left(\varphi\left[\left\{f_{1}, \ldots, f_{p-1}, f_{p}^{\otimes 2}, \theta f_{p-1}, \ldots, \theta f_{1}\right\}\right]\right)
\end{aligned}
$$

If we now regard $\left(\theta f_{p+1}\right)^{\otimes 2}$ and $f_{p}^{\otimes 2}$ as two new functions in $H_{0}^{1}\left(\left[0,2 T_{p+1}\right]\right)$ and in $H_{0}^{1}\left(\left[0,2 T_{p}\right]\right)$, respectively, the two terms in the r.h.s. of (3.18) can be regarded as two terms with $N=2 p-1$ and, by the induction assumption, they satisfy the bounds:

$$
\begin{aligned}
& \mathcal{E}_{0,2\left(x_{2 p}-x_{p}\right)}^{\mathrm{D}}\left(\varphi\left[\left\{\theta f_{2 p}, \ldots, \theta f_{p+2},\left(\theta f_{p+1}\right)^{\otimes 2}, f_{p+2}, \ldots f_{2 p}\right\}\right]\right) \geq 2 \sum_{i=p+1}^{2 p} T_{i} e_{\infty}\left(f_{i}\right), \\
& \mathcal{E}_{0,2 x_{p}}^{\mathrm{D}}\left(\varphi\left[\left\{f_{1}, \ldots, f_{p-1}, f_{p}^{\otimes 2}, \theta f_{p-1}, \ldots, \theta f_{1}\right\}\right]\right) \geq 2 \sum_{i=1}^{p} T_{i} e_{\infty}\left(f_{i}\right)
\end{aligned}
$$

where we used that $e_{\infty}\left(\left(\theta f_{p+1}\right)^{\otimes 2}\right)=e_{\infty}\left(f_{p+1}\right)$ and $e_{\infty}\left(f_{p}^{\otimes 2}\right)=e_{\infty}\left(f_{p}\right)$. Therefore, the desired bound is proved.

(b) $n=2 p+1$ for some $p \geq 1$. If we reflect once we get:

$$
\begin{aligned}
& \mathcal{E}_{0, x_{2 p+1}}^{\mathrm{D}}\left(\varphi\left[\left\{f_{1}, \ldots, f_{2 p+1}\right\}\right]\right) \geq \\
& \geq \frac{1}{2} \mathcal{E}_{0,2\left(x_{2 p+1}-x_{p+1}\right)}^{\mathrm{D}}\left(\varphi\left[\left\{\theta f_{2 p+1}, \ldots, \theta f_{p+3},\left(\theta f_{p+2}\right)^{\otimes 2}, f_{p+3}, \ldots, f_{2 p+1}\right\}\right]\right)+ \\
& +\frac{1}{2} \mathcal{E}_{0,2 x_{p+1}}^{\mathrm{D}}\left(\varphi\left[\left\{f_{1}, \ldots, f_{p}, f_{p+1}^{\otimes 2}, \theta f_{p}, \ldots, \theta f_{1}\right\}\right]\right)
\end{aligned}
$$

The first term in the r.h.s. corresponds to $N=2 p-1$ so by the induction hypothesis it is bounded below by $\sum_{i=p+2}^{2 p+1} T_{i} e_{\infty}\left(f_{i}\right)$. As regards the second term, using reflection positivity again, we can bound it from below by

$$
\frac{1}{4} \mathcal{E}_{0,2 x_{p}}^{\mathrm{D}}\left(\varphi\left[\left\{f_{1}, \ldots, f_{p}, \theta f_{p}, \ldots, \theta f_{1}\right\}\right]\right)+\frac{1}{4} \mathcal{E}_{0,2 x_{p}+4 x_{p+1}}^{\mathrm{D}}\left(\varphi\left[\left\{f_{1}, \ldots, f_{p},\left(f_{p+1}\right)^{\otimes 4}, \theta f_{p}, \ldots, \theta f_{1}\right\}\right]\right)
$$

By the induction hypothesis, the first term is bounded below by $(1 / 2) \sum_{i=1}^{p} T_{i} e_{\infty}\left(f_{i}\right)$, and the second can be bounded using reflection positivity again. Iterating we find:

$$
\begin{aligned}
& \mathcal{E}^{\mathrm{D}}\left(\varphi\left[\left\{f_{1}, \ldots, f_{2 p+1}\right\}\right]\right) \geq \\
& \left.\geq \sum_{i=p+2}^{2 p+1} T_{i} e_{\infty}\left(f_{i}\right)+\left(\sum_{n \geq 1} 2^{-n}\right) \cdot \sum_{i=1}^{p} T_{i} e_{\infty}\left(f_{i}\right)\right)+ \\
& \quad+\lim _{n \rightarrow \infty} 2^{-n} \mathcal{E}_{0,2 x_{p}+2^{m}}^{\mathrm{D}} x_{p+1}\left(\varphi\left[\left\{f_{1}, \ldots, f_{p},\left(f_{p+1}\right)^{\otimes 2^{m}}, \theta f_{p}, \ldots, \theta f_{1}\right\}\right]\right) .
\end{aligned}
$$


Note that the last term is equal to $T_{p+1} e_{\infty}\left(f_{p+1}\right)$, so $(3.22)$ is the desired bound. This concludes the proof of (3.12).

Proof of Theorem 1. (1) Let us first prove that $e_{0}=\inf _{T} \inf _{f \in \mathcal{C}_{T}} e_{\infty}(f)$. First of all, let us note that $\lim \sup _{L \rightarrow \infty} E_{L}^{\mathrm{F}} / L=\limsup _{L \rightarrow \infty} E_{L}^{\mathrm{D}} / L$ and $\liminf _{L \rightarrow \infty} E_{L}^{\mathrm{F}} / L=\liminf _{L \rightarrow \infty} E_{L}^{\mathrm{D}} / L$, because the interaction $v$ is absolutely summable. Moreover, it follows by the variational estimate $E_{L}^{\mathrm{F}} \leq \mathcal{E}_{0, L}^{\mathrm{F}}(\varphi[f])$, valid for any $f \in \mathcal{C}_{T}, T>0$, that $\lim _{\sup _{L \rightarrow \infty}} E_{L}^{\mathrm{F}} / L \leq$ $\inf _{T} \inf _{f \in \mathcal{C}_{T}} e_{\infty}(f)$.

We then just need to prove that $\liminf _{L \rightarrow \infty} E_{L}^{\mathrm{D}} / L \geq \inf _{T} \inf _{f \in \mathcal{C}_{T}} e_{\infty}(f)$. For this purpose, given $L>0$, let $\phi$ be any function in $H_{0}^{1}([0, L])$ and let us denote by $x_{0}=0<x_{1}<\ldots<x_{N}=L$ its nodes (if $\phi$ is identically 0 in some interval $[a, b] \subseteq[0, L]$, it will be understood that $\phi$ has two nodes between $a$ and $b$, the first located at $x=a$ the second at $x=b)$. We define: $T_{i}=x_{i}-x_{i-1}$, $i=1, \ldots, N$. Let $f_{i}:\left[0, T_{i}\right] \rightarrow \mathbb{R}$ be such that $\phi(x)=f_{i}\left(x-x_{i-1}\right)$, for all $x \in\left[x_{i-1}, x_{i}\right]$. By construction any $f_{i}$ is either nonnegative or nonpositive and $\phi=\varphi\left[\left\{f_{1}, \ldots, f_{N}\right\}\right]$. Using the chessboard estimate we find:

$$
\mathcal{E}_{0, L}^{\mathrm{D}}(\phi)=\mathcal{E}_{0, L}^{\mathrm{D}}\left(\varphi\left[\left\{f_{1}, \ldots, f_{N}\right\}\right]\right) \geq \sum_{i=1}^{N} T_{i} e_{\infty}\left(f_{i}\right)
$$

All the $e_{\infty}\left(f_{i}\right)$ in the r.h.s. can be bounded below by $\inf _{T} \inf _{f \in \mathcal{C}_{T}} e_{\infty}(f)$, and the proof of this first claim is concluded.

(2) Next, let us show that for any fixed $T>0$ there exists a function $\phi_{T} \in \mathcal{C}_{T}$ such that $\left|\phi_{T}\right| \leq 1$ and $e_{\infty}\left(\phi_{T}\right)=\inf _{f \in \mathcal{C}_{T}} e_{\infty}(f) \equiv e_{T}$. Note that, for any $f \in \mathcal{C}_{T}, e_{\infty}(f)$ can be rewritten as:

$$
e_{\infty}(f)=\frac{1}{T} \int_{0}^{T} d x\left(f^{\prime}(x)\right)^{2}+\frac{1}{T} \int_{0}^{T} d x F(f(x))+\frac{1}{T} \int_{0}^{T} d x \int_{0}^{T} d y f(x) f(y) \widetilde{v}_{T}(x, y)
$$

with

$$
\widetilde{v}_{T}(x, y)=\sum_{n \in \mathbb{Z}}[v(2 n T+y-x)-v(y+x+2 n T)]
$$

Since $F \geq 0$, the first two terms on the r.h.s. of (3.24) are clearly nonnegative. Since $v$ is absolutely summable, the potential $\widetilde{v}_{T}(x, y)$ can be rewritten as

$$
\widetilde{v}_{T}(x, y)=\sum_{n \geq 0}[v(2 n T+y-x)-v(2(n+1) T-y-x)-v(2 n T+y+x)+v(2(n+1) T-y+x)]
$$


and, using the fact that $v^{\prime \prime} \geq 0$, it is straightforward to check that each term in the sum on the r.h.s. is pointwise positive, for all $0 \leq x, y \leq T$. This implies that the third term in the r.h.s. of (3.24) is nonnegative as well. We will denote the kinetic energy, i.e., the first term in the r.h.s. of (3.24), by $K_{f}$, and the second and third terms by $V_{f}$ and $W_{f}$, respectively.

Now let $f^{j}$ be a minimizing sequence, i.e., $e_{\infty}\left(f^{j}\right) \rightarrow e_{T}$ as $j \rightarrow \infty$ and $f^{j} \in \mathcal{C}_{T}$. First we note that $K_{f^{j}}$ is bounded by a constant independent of $j$, because $K_{f^{j}} \leq T e_{\infty}\left(f^{j}\right)$ and $e_{\infty}\left(f^{j}\right)$ is uniformly bounded from above by some constant $C$. Moreover, we can assume without loss of generality that $\left|f^{j}\right| \leq 1$ : in fact, since $F(t)$ has an absolute minimum at $t=1$ and the potential $\widetilde{v}(x, y)$ is pointwise positive, we see that the energy in (3.24) decreases by replacing $f$ with $\min \{f, 1\}$. In fact, each of the three terms in the energy can only decrease with the replacement. Then the sequence $f^{j}$ is bounded in $H_{0}^{1}([0, T])$. Since bounded sets in $H_{0}^{1}([0, T])$ are weakly sequentially compact (see [21], Section 7.18), we can therefore find a function $\phi_{T}$ in $H_{0}^{1}([0, T])$ and a subsequence (which we continue to denote by $f^{j}$ ) such that $f^{j} \rightarrow \phi_{T}$ weakly in $H_{0}^{1}([0, T])$. By Corollary 8.7 in [21] ("weak convergence implies a.e. convergence"), we can assume without loss of generality that $f^{j}(x) \rightarrow \phi_{T}(x)$ for almost every $x \in[0, T]$. This function $\phi_{T}$ satisfies $\left|\phi_{T}\right| \leq 1$ and will be our minimizer: in fact, since the kinetic energy $K_{f}$ is weakly lower semicontinuous (see [21], Section 8.2), and since $V_{f^{j}} \rightarrow V_{\phi_{T}}$ and $W_{f^{j}} \rightarrow W_{\phi_{T}}$ as $j \rightarrow \infty$, by the dominated convergence theorem, we have that

$$
e_{T}=\lim _{j \rightarrow \infty} e_{\infty}\left(f_{j}\right) \geq e_{\infty}\left(\phi_{T}\right)
$$

and this shows that $\phi_{T}$ is the minimizer.

(3) Now, let us show that $e_{T}$ is continuous in $T$. We shall do this by deriving bounds from above and below on $e_{T+\varepsilon}$, tending to $e_{T}$ as $\varepsilon \rightarrow 0$. Let us take $\varepsilon>0$. In order to get the bound from above on $e_{T+\varepsilon}$, let us consider a variational function $f_{T+\varepsilon} \in H_{0}^{1}([0, T+\varepsilon])$, coinciding with $\phi_{T}$, i.e., the minimizer of $e_{T}$, for $x \in[0, T]$, and equal to 0 in $x \in[T, T+\varepsilon]$. Using (3.24) we get

$$
\begin{aligned}
e_{T+\varepsilon} \leq e_{\infty}\left(f_{T+\varepsilon}\right) & =\frac{1}{T+\varepsilon} \int_{0}^{T} d x\left(\phi_{T}^{\prime}(x)\right)^{2}+\frac{1}{T+\varepsilon} \int_{0}^{T} d x F\left(\phi_{T}(x)\right)+\frac{\varepsilon}{T+\varepsilon} F(0)+ \\
& +\frac{1}{T+\varepsilon} \int_{0}^{T} d x \int_{0}^{T} d y \phi_{T}(x) \phi_{T}(y) \widetilde{v}_{T+\varepsilon}(x, y)
\end{aligned}
$$


and clearly $e_{\infty}\left(f_{T+\varepsilon}\right) \rightarrow e_{\infty}\left(\phi_{T}\right)$ as $\varepsilon \rightarrow 0$. In order to get a lower bound, let us use the variational estimate $e_{T} \leq e_{\infty}\left(g_{T}\right)$, where $g_{T}=\phi_{T+\varepsilon}(x(1+\varepsilon / T))$. Using (3.24), we get

$$
\begin{aligned}
e_{T} \leq e_{\infty}\left(g_{T}\right)=\frac{1}{T}(1 & \left.+\frac{\varepsilon}{T}\right) \int_{0}^{T+\varepsilon} d x\left[\phi_{T+\varepsilon}^{\prime}(x)\right]^{2}+\frac{1}{T}\left(1+\frac{\varepsilon}{T}\right)^{-1} \int_{0}^{T+\varepsilon} d x F\left(\phi_{T+\varepsilon}(x)\right)+ \\
+\frac{1}{T}(1 & \left.+\frac{\varepsilon}{T}\right)^{-2} \int_{0}^{T+\varepsilon} d x \int_{0}^{T+\varepsilon} d y \phi_{T+\varepsilon}(x) \phi_{T+\varepsilon}(y) \times \\
& \times \sum_{n \in \mathbb{Z}}\left[v\left(\frac{2 n(T+\varepsilon)+y-x}{1+\varepsilon / T}\right)-v\left(\frac{2 n(T+\varepsilon)+y+x}{1+\varepsilon / T}\right)\right]
\end{aligned}
$$

and clearly $e_{\infty}\left(g_{T}\right)-e_{\infty}\left(\phi_{T+\varepsilon}\right) \rightarrow 0$ as $\varepsilon \rightarrow 0$. A similar proof applies to the case $\varepsilon<0$, therefore continuity of $e_{T}$ is proved.

(4) Let us prove that $\lim _{T \rightarrow \infty} e_{T}$ exists and is equal to:

$$
\lim _{T \rightarrow \infty} e_{T}=\inf _{f \in \mathcal{C}_{\infty}} \liminf _{L \rightarrow \infty} \frac{\mathcal{E}_{-L, L}^{\mathrm{F}}(f)}{2 L}
$$

where $\mathcal{C}_{\infty}=\left\{f \in H_{\text {loc }}^{1}(\mathbb{R}): 0 \leq f \leq 1\right\}$. For this purpose, if $\phi_{T}$ is the minimizer of $e_{T}$, let us rewrite

$$
\begin{aligned}
& e_{\infty}\left(\phi_{T}\right)=\frac{1}{T} \int_{0}^{T} d x\left[\left(\phi_{T}^{\prime}(x)\right)^{2}+F\left(\phi_{T}(x)\right)\right]+\frac{1}{T} \int_{0}^{T} d x \int_{0}^{T} d y \phi_{T}(x) \phi_{T}(y) v(y-x)+ \\
& +\frac{1}{T} \int_{0}^{T} d x \int_{0}^{T} d y \phi_{T}(x) \phi_{T}(y) \sum_{n \geq 1}[v(2 n T+y-x)-v(2 n T-y-x)]+ \\
& +\frac{1}{T} \int_{0}^{T} d x \int_{0}^{T} d y \phi_{T}(x) \phi_{T}(y) \sum_{n \geq 1}[v(2 n T-y+x)-v(2(n-1) T+y+x)] .
\end{aligned}
$$

Using the fact that $0 \leq \phi_{T} \leq 1$, as proved in part $(2)$, and the fact that $v \in L^{1}(\mathbb{R}) \cap L^{\infty}(\mathbb{R})$ is completely monotone, we find that the last two terms in (3.31) tend to 0 as $T \rightarrow \infty$. Therefore:

$$
e_{T}=\frac{\mathcal{E}_{0, T}^{\mathrm{D}}\left(\phi_{T}\right)}{T}+o(1)=\inf _{f \in \mathcal{C}_{T}^{*}} \frac{\mathcal{E}_{0, T}^{\mathrm{F}}(f)}{T}+o(1)
$$

where $\mathcal{C}_{T}^{*}=\left\{f \in H^{1}([0, T]): 0 \leq f \leq 1\right\}$. Repeating the proof in part (2), we find that the inf in the r.h.s. is a minimum, and we denote by $f_{T}$ the corresponding minimizer. Note that the quantity $\mathcal{E}_{0, T}^{\mathrm{F}}\left(f_{T}\right)$ is superadditive in $T$, i.e., $\mathcal{E}_{0, T_{1}+T_{2}}^{\mathrm{F}}\left(f_{T_{1}+T_{2}}\right) \geq \mathcal{E}_{0, T_{1}}^{\mathrm{F}}\left(f_{T_{1}}\right)+\mathcal{E}_{0, T_{2}}^{\mathrm{F}}\left(f_{T_{2}}\right)$. Then the limit as $T \rightarrow \infty$ of $\mathcal{E}_{0, T}^{\mathrm{F}}\left(f_{T}\right) / T$ exists and

$$
\lim _{T \rightarrow \infty} e_{T}=\lim _{T \rightarrow \infty} \frac{\mathcal{E}_{0, T}^{\mathrm{F}}\left(f_{T}\right)}{T} .
$$

Now, on the one hand, using the variational estimate $\mathcal{E}_{0, T}^{\mathrm{F}}\left(f_{T}\right) \leq \mathcal{E}_{0, T}^{\mathrm{F}}(f)$ valid for any $f \in \mathcal{C}_{\infty}$ we see that the limit on the r.h.s. of (3.33) is smaller than $\inf _{f \in \mathcal{C}_{\infty}} \liminf _{L \rightarrow \infty} \mathcal{E}_{-L, L}^{\mathrm{F}}(f) /(2 L)$. 
On the other hand, using summability and complete monotonicity of $v$, we find that for any $T$

$$
\frac{\mathcal{E}_{0, T}^{\mathrm{F}}\left(f_{T}\right)}{T}=\lim _{L \rightarrow \infty} \frac{\mathcal{E}_{-L, L}^{\mathrm{F}}\left(\widetilde{\varphi}\left[f_{T}\right]\right)}{2 L}+o(1)
$$

where $\widetilde{\varphi}\left(\left[f_{T}\right]\right) \in \mathcal{C}_{\infty}$ is the function obtained by periodically repeating the sequence $\left\{f_{T},-\theta f_{T}\right\}$ infinitely many times and $o(1)$ is a remainder that goes to 0 as $T \rightarrow \infty$. Clearly, the first term in the r.h.s. can be bounded from below by $\inf _{f \in \mathcal{C}_{\infty}} \liminf _{L \rightarrow \infty} \mathcal{E}_{-L, L}^{\mathrm{F}}(f) /(2 L)$ and this concludes the proof of the claim.

(5) Finally, it is straightforward to check that if the distributional derivative of $F$ is a function, then the minimizer $\phi_{T}$ satisfies the Euler-Lagrange equation in the sense of distributions. If $F(t)$ is differentiable for $t>0$, by the smoothness of $v$, it follows by a standard "bootstrap" argument (see Theorem 11.7 in [21]), that $\phi_{T} \in C^{2}$.

If $F(t)$ is convex for $t>0$, then the functional $e_{\infty}\left(f_{T}\right)$ is strictly convex (because $\int\left(f^{\prime}\right)^{2}$ is strictly convex) for $f_{T} \in \mathcal{C}_{T}$ and the minimizer $\phi_{T}$ is unique. Similarly, for any $L>0$, the functional $\mathcal{E}_{0, L}^{\mathrm{F}}(f) / L$ is strictly convex for $f \in \mathcal{C}_{L}^{*}$ and so is $\mathcal{E}_{0, L}^{\text {per }}(f) / L$, where $\mathcal{E}_{0, L}^{\text {per }}(f)$ is the analogue of $\mathcal{E}_{0, L}^{\mathrm{F}}(f)$ with periodic boundary conditions:

$$
\mathcal{E}_{0, L}^{\mathrm{per}}(f)=\int_{0}^{L} d x\left[\left(f^{\prime}(x)\right)^{2}+F(f)\right]+\int_{0}^{L} d x \int_{0}^{L} d y f(x) f(y) \sum_{n \in \mathbb{Z}} v(n L+y-x)
$$

with $f \in \mathcal{C}_{L}^{\text {per }} \equiv\left\{g \in \mathcal{C}_{L}^{*}: g(0)=g(L)\right\}$.

By the summability of $v$,

$$
\liminf _{L \rightarrow \infty} \frac{\mathcal{E}_{0, L}^{\mathrm{F}}(f)}{L}=\liminf _{L \rightarrow \infty} \frac{\mathcal{E}_{0, L}^{\mathrm{per}}(f)}{L}, \quad \forall f \in \mathcal{C}_{\infty}
$$

By periodicity, $\mathcal{E}_{0, L}^{\text {per }}(f)=\frac{1}{L} \int_{0}^{L} \mathcal{E}_{0, L}^{\text {per }}\left(\tau_{x} f\right)$, where $\tau_{x} f(y) \equiv f(y-x)$. By convexity, the latter quantity is bounded below by $\mathcal{E}_{0, L}^{\mathrm{per}}(\langle f\rangle)=L\left[F(\langle f\rangle)+\langle f\rangle^{2} \int_{0}^{L} \frac{d x}{L} \int_{0}^{L} d y v(x-y)\right]$. This shows that the limit as $T \rightarrow \infty$ of $e_{T}$ is $\min _{t \in \mathbb{R}^{+}}\left\{F(t)+t^{2} \int_{-\infty}^{+\infty} d x v(x)\right\}$ and concludes the proof of Theorem 1.

Corollary 1 is a simple consequence of Theorem 1 and of its proof.

Proof of Corollary 1. (i). Let $T_{0}$ be such that $e_{0}=e_{T_{0}}$ and let us assume by contradiction that there exists an interval $[a, b]$ and a function $f \in H_{l o c}^{1}(\mathbb{R})$, coinciding with 
$\varphi\left[\phi_{T_{0}}\right]$ on $\mathbb{R} \backslash[a, b]$, and such that $\mathcal{E}_{a, b}(f)<\mathcal{E}_{a, b}\left(\varphi\left[\phi_{T_{0}}\right]\right)$. Note that, if $\left[a^{\prime}, b^{\prime}\right] \supseteq[a, b]$, then $\mathcal{E}_{a^{\prime}, b^{\prime}}(f)-\mathcal{E}_{a^{\prime}, b^{\prime}}\left(\varphi\left[\phi_{T_{0}}\right]\right)=\mathcal{E}_{a, b}(f)-\mathcal{E}_{a, b}\left(\varphi\left[\phi_{T_{0}}\right]\right)$. We choose $\left[a^{\prime}, b^{\prime}\right] \supseteq[a, b]$ such that $b^{\prime}-a^{\prime}=k T_{0}$, for some $k \in \mathbb{N}$, and $f\left(a^{\prime}\right)=f\left(b^{\prime}\right)=0$. We denote by $f_{1}$ the restriction of $f$ to $\left[a^{\prime}, b^{\prime}\right]$ and we write:

$$
\begin{aligned}
0> & \mathcal{E}_{a, b}(f)-\mathcal{E}_{a, b}\left(\varphi\left[\phi_{T_{0}}\right]\right) \\
& =\lim _{m \rightarrow \infty}\left[\mathcal{E}_{a^{\prime}-m T_{0}, b^{\prime}+m T_{0}}^{\mathrm{D}}\left(\varphi\left[\left\{\phi_{T_{0}}^{\otimes m}, f_{1}, \phi_{T_{0}}^{\otimes m}\right\}\right]\right)-\mathcal{E}_{a^{\prime}-m T_{0}, b^{\prime}+m T_{0}}^{\mathrm{D}}\left(\varphi\left[\phi_{T_{0}}\right]\right)\right] \\
& =\lim _{m \rightarrow \infty}\left[\mathcal{E}_{a^{\prime}-m T_{0}, b^{\prime}+m T_{0}}^{\mathrm{per}}\left(\varphi\left[\left\{\phi_{T_{0}}^{\otimes m}, f_{1}, \phi_{T_{0}}^{\otimes m}\right\}\right]\right)-\mathcal{E}_{a^{\prime}-m T_{0}, b^{\prime}+m T_{0}}^{\mathrm{per}}\left(\varphi\left[\phi_{T_{0}}\right]\right)\right] .
\end{aligned}
$$

Now, $\mathcal{E}_{a^{\prime}-m T_{0}, b^{\prime}+m T_{0}}^{\text {per }}\left(\varphi\left[\phi_{T_{0}}\right]\right)=(2 m+k) T_{0} e_{0}$ and, by the chessboard inequality,

$$
\mathcal{E}_{a^{\prime}-m T_{0}, b^{\prime}+m T_{0}}^{\text {per }}\left(\varphi\left[\left\{\phi_{T_{0}}^{\otimes m}, f_{1}, \phi_{T_{0}}^{\otimes m}\right\}\right]\right) \geq 2 m T_{0} e_{0}+k T_{0} e_{\infty}\left(f_{1}\right)
$$

so that we find $e_{\infty}\left(f_{1}\right)-e_{0}<0$, which is a contradiction.

(ii) Let $e_{0}=\lim _{T \rightarrow \infty} e_{T}$ and $F(t)$ convex for $t>0$. As proved in Theorem 1,

$e_{0}=\min _{t \in \mathbb{R}^{+}}\left\{F(t)+t^{2} \int_{-\infty}^{+\infty} d x v(x)\right\} \equiv F\left(t_{0}\right)+t_{0}^{2} \int_{-\infty}^{+\infty} d x v(x)$ and a repetition of the proof in part (i) shows that $f(x) \equiv t_{0}$ is an infinite volume ground state.

\section{AN EXAMPLE}

One expects that when $v(x) x$ is summable, i.e., $\int_{0}^{\infty} \nu(d \alpha) \alpha^{-2}<+\infty$, the minimizer is periodic when $\lambda=v(0)$ is large, while small $\lambda$ produces a function with constant sign, say positive. If $v(x) x$ is not summable one expects that the minimizer is always periodic. We shall not prove this last statement, but see [17] for a similar discussion in the discrete microscopic case.

Here we give an illustrative example that will make this small $\lambda$, large $\lambda$ dichotomy clear. This example is generic, in the convex case, at least, it is only a question of estimating orders of magnitude in the two regimes of $\lambda$.

Let $v(x)=\lambda e^{-|x|}$ and $F(\phi)=(|\phi|-1)^{2}$. This is the "convex case", in the sense that $F^{\prime \prime}(\phi)>0$ for $\phi>0$. When $\lambda=0$ the minimum energy occurs when $\phi^{\prime}=0$ and $F=0$, which means that either $\phi(x)=1$ for all $x$ or $\phi=-1$ for all $x$. For small $\lambda, \phi$ will be of constant sign, 
and hence a constant, by convexity. To see this it suffices to note that $|\phi(x)|$ must be nearly 1 for most $x$ (by continuity of the energy), and if $\phi$ had a jump from +1 to -1 the cost in kinetic energy $\int\left|\phi^{\prime}\right|^{2}$ would outweigh any gain in the integral term coming from the interaction of a negative $\phi$ region and a positive $\phi$ region - which would be of the order of $\lambda$, at best.

To show that one gets periodicity for large $\lambda$ it is only necessary to write down the energy for the constant $\phi$ case and compare it with a crude variational periodic $\phi$. The constant is easily calculated to be $\phi_{0}=(1+2 \lambda)^{-1} \ll 1$ and the specific energy is $e=2 \lambda(1+2 \lambda)^{-1}$. The variational function can be taken to be $\pm \phi_{0}$ with a large period $T$ and with a linear interpolation between $+\phi_{0}$ and $-\phi_{0}$ of width $\beta \sim \lambda^{-1 / 2} \ll 1$. This gives a local energy (i.e., the first term in $(2.1)) \sim \lambda^{-3 / 2}$ for each such interface. The gain in interaction energy across the interface is $\sim-\lambda^{-1}$, which is greater than this.

There is no need to belabor the details of such examples. The conclusion is that there must be a transition from constant to periodic as $\lambda$ increases. The critical $\lambda_{c}$ at which the transition occurs can be computed by imposing the condition that the energy of the "kink", i.e., the antisymmetric solution to the Euler-Lagrange equation with boundary conditions $\phi( \pm \infty)=$ $\pm \phi_{0}$ and $\phi(0)=0$, is the same as that of the constant function $\phi(x) \equiv \phi_{0}$ (note that both energies are infinite, but the energy difference is well defined and finite). In our example the kink solution $\phi$ can be computed exactly, and likewise its energy. To be specific, let us write the Euler-Lagrange equation for $\phi(x), x \geq 0$, as:

$$
-\phi^{\prime \prime}(x)+\phi(x)-1+\lambda \int_{0}^{\infty} d y\left(e^{-|x-y|}-e^{-x-y}\right) \phi(y)=0 .
$$

Defining $h(x)=\int_{0}^{\infty} d y e^{-|x-y|} \phi(y)$ and $c=\int_{0}^{\infty} d y e^{-y} \phi(y)$, we can rewrite this equation as

$$
-\phi^{\prime \prime}(x)+\phi(x)-1+\lambda h(x)-\lambda c e^{-x}=0 .
$$

This implies that $\phi^{\prime \prime}(0)=-1$. If we differentiate twice and use the fact that $h^{\prime \prime}(x)=h(x)-$ $2 \phi(x)$, we find:

$$
\begin{aligned}
& -\phi^{\prime \prime \prime \prime}(x)+\phi^{\prime \prime}(x)+\lambda h(x)-2 \lambda \phi(x)-\lambda c e^{-x}= \\
& =\quad-\phi^{\prime \prime \prime \prime}(x)+2 \phi^{\prime \prime}(x)-(1+2 \lambda) \phi(x)+1=0,
\end{aligned}
$$

where we used (4.40) to rewrite $\lambda h(x)-\lambda c e^{-x}=\phi^{\prime \prime}(x)-\phi(x)+1$. The only solution to (4.41) 
satisfying $\phi(0)=0, \phi(+\infty)=\phi_{0}=(1+2 \lambda)^{-1}$ and $\phi^{\prime \prime}(0)=-1$ is

$$
\phi(x)=\frac{1}{1+2 \lambda}\left(1-e^{-\mu_{1} x} \frac{\cos \left(\mu_{2} x+\theta\right)}{\cos \theta}\right),
$$

where:

$$
\mu=\mu_{1}+i \mu_{2}=(1+2 \lambda)^{1 / 4} e^{i \theta / 2} \quad \text { and } \quad \theta=\arcsin \sqrt{\frac{2 \lambda}{1+2 \lambda}}
$$

The difference between the energy of the solution in (4.42) and that of the constant function $\phi(x)=(1+2 \lambda)^{-1}$ is

$$
\Delta E=\frac{2}{1+2 \lambda} \int_{0}^{\infty} d x e^{-\mu_{1} x} \frac{\cos \left(\mu_{2} x+\theta\right)}{\cos \theta}
$$

and imposing $\Delta E=0$ we get the condition $\cos (3 \theta / 2)=0$, which is equivalent to

$$
\lambda=\lambda_{c}=\frac{3}{2} .
$$

The conclusion is that in our example the infinite volume ground state is the constant function $\phi(x)=(1+2 \lambda)^{-1}$, for all $\lambda \leq 3 / 2$, and is periodic, for all $\lambda>3 / 2$.

\section{DISCUSSION AND CONNECTION WITH RELATED WORK}

We investigated a class of 1D free-energy functionals, characterized by a competition between a local term, prefering a constant minimizer (equal to 1 or -1 , the positions of the minima of a "double-well" even function $F$ ), and a long range positive interaction, which is assumed to be reflection positive and summable. We showed by reflection positivity that, for any strength of the long range interaction, the ground state is either periodic (with mean zero) or of constant sign. If the local term $F(\phi)$, besides being even in $\phi$, is assumed to be convex on $\mathbb{R}^{+}$, then the ground state is either periodic or constant. The proof is simple and does not depend on the details of the function $F$ or $v$ (as long as $v$ is positive and reflection positive). Note, however, that the assumption that $F$ is even is crucial: this means that we cannot include a chemical potential different from zero.

Our results extend or complement some known results first proved by Alberti and Müller [2] and by Chen and Oshita [9] on periodicity of the minimizers of certain 1D free-energy 
functionals. They consider models similar to (2.1), with non-zero chemical potential. They require however a smallness condition on the gradient term and there are certain classes of potentials, e.g., power law type interactions, which are not included in their proofs. Their analysis is based on detailed apriori asymptotic estimates that are not needed in our approach. Let us conclude by mentioning the connection of our results with the so-called "froth problem" put forward by Lebowitz and Penrose in [22]. They consider $d$-dimensional systems of particles with density $\rho$ (or spin systems with magnetization $m$ ) interacting both with a short range interaction and with a long range Kac potential of the form $\gamma^{d} v(\gamma \mathbf{r})$, with $\int_{\mathbb{R}^{d}} v(\mathbf{r}) d \mathbf{r}=\alpha$. When $\gamma \rightarrow 0$, the exact free energy per unit volume, $a(\rho)$, for the case where $v$ is positive definite (which includes the cases considered here) is given by $a(\rho)=a_{s}(\rho)+\frac{1}{2} \alpha \rho^{2} ; a_{s}(\rho)$ is the free energy due to the short range potential. In cases where the short range interaction induces a phase separation, as indicated by a linear segment in $a_{s}(\rho)$, the long range positive definite Kac potential, with $\alpha>0$, will lead to a strictly convex $a(\rho)$. This means that the global phase segregation, due to the short range interaction, is destroyed by the long range positive definite Kac potential in the limit $\gamma \rightarrow 0$. The interpretation given in [22] was that there is no phase separation on the scale $\gamma^{-1}$, but non trivial structures may appear on an intermediate scale $1 \ll \gamma^{-\delta} \ll \gamma^{-1}$. In this sense the system for finite, but small, $\gamma$ is expected to be a sort of froth, with structures invisible on large scales, but observable on intermediate scales (and these structures may form periodic patterns, as discussed in the introduction). While the scale $\gamma^{-\delta}$ is unknown in general, our results on microscopic models show that, at least in 1D lattice models, the correct scale to look at, at zero temperature, is $\gamma^{-2 / 3}$, see [17] and the discussion in Section VIII of [18].

The problem of understanding these mesoscopic structures can be related to the minimization problem studied in this paper, thanks to a result by Gates and Penrose [15], who proved that the free energy $a(\rho)$ can also be obtained from a minimization of a free energy functional similar to (2.1), but without a gradient term. Such functional has the interpretation of large deviation functional for observing a mesoscopic density $[3,4,8,11]$. As already noted, the absence of a gradient term in this functional means that its minimizers (the "typical mesoscopic configurations") will oscillate on a scale small compared to $\gamma^{-1}$, e.g., the $\gamma^{-2 / 3}$ found in [18]. 
It would be nice to understand the correspondence between these oscillations for $\gamma \ll 1$ and the ones found here and in [2].

\section{Acknowledgments}

We thank Paolo Buttà, Anna De Masi, Errico Presutti and particularly Eric Carlen for valuable discussions. The work of JLL was supported by NSF Grant DMR-044-2066 and by AFOSR Grant AF-FA 9550-04-4-22910. The work of AG and EHL was partially supported by U.S. National Science Foundation grant PHY-0652854.

[1] J. Arlett, J. P. Whitehead, A. B. MacIsaac, and K. De'Bell: Phase diagram for the striped phase in the two-dimensional dipolar Ising model Phys. Rev. B 54, 3394 (1996).

[2] G. Alberti and S. Müller: A new approach to variational problems with multiple scales, Communications on Pure and Applied Mathematics 54, 761-825 (2001).

[3] O. Benois, T. Bodineau, P. Buttà, and E. Presutti: On the validity of van der Waals theory of surface tension, Markov Process. Related Fields 3, 175-198 (1997).

[4] O. Benois, T. Bodineau and E. Presutti: Large deviations in the van der Waals limit, Stochastic Process. Appl. 75, 89-104 (1998).

[5] V. Y. Borue and I. Y. Erukhimovich: A Statistical Theory of Weakly Charged Polyelectrolytes: Fluctuations, Equation of State, and Microphase Separation, Macromolecules 21, 3240 (1988).

[6] P. Buttà and J. L. Lebowitz: Local Mean Field Models of Uniform to Nonuniform Density (fluidcrystal) Transitions Journal of Physical Chemistry B 109, 6849-6854 (2005).

[7] S. A. Brazovskii: Phase transition of an isotropic system to a non uniform state Zh. Eksp. Teor. Fiz. 68, 175 (1975).

[8] E. Carlen, M. C. Carvalho, R. Esposito, J. L. Lebowitz and R. Marra: Phase Transitions in Equilibrium Systems: Microscopic Models and Mesoscopic Free Energies, Journal of Molecular Physics 103, 3141-3151 (2005).

[9] X. Chen and Y. Oshita: Periodicity and Uniqueness of Global Minimizers of an Energy Functional Containing a Long-Range Interaction, SIAM Journal on Mathematical Analysis 37, 1299-1332 (2006). 
[10] A. DeSimone, R. V. Kohn, F. Otto and S. Müller: Recent analytical developments in micromagnetics, in The Science of Hysteresis II: Physical Modeling, Micromagnetics, and Magnetization Dynamics, G. Bertotti and I. Mayergoyz eds., pp. 269-381, Elsevier (2001).

[11] P. Dupuis and R. S. Ellis: A weak convergence approach to the theory of large deviations, Wiley Series in Probability and Statistics, John Wiley \& Sons, Inc., New York (1997).

[12] V. J. Emery and S. A. Kivelson: Frustrated electronic phase separation and high-temperature superconductors, Physica C 209, 597 (1993).

[13] J. Frohlich, R. Israel, E. H. Lieb and B. Simon: Phase Transitions and Reflection Positivity. I. General Theory and Long Range Lattice Models, Communications in Mathematical Physics 62, 1 (1978).

[14] T. Garel and S. Doniach: Phase transitions with spontaneous modulation-the dipolar Ising ferromagnet Phys. Rev. B 26, 325 (1982).

[15] D. J. Gates and O. Penrose: The van der Waals limit for classical systems. I. A variational principle, Communications in Mathematical Physics 15, 255-276 (1969).

[16] D. J. Gates and O. Penrose: The van der Waals limit for classical systems. III. Deviation from the van der Waals-Maxwell theory, Communications in Mathematical Physics 17, 194-209 (1970).

[17] A. Giuliani, J. L. Lebowitz and E. H. Lieb: Ising models with long-range dipolar and short range ferromagnetic interactions, Physical Review B 74, 064420 (2006).

[18] A. Giuliani, J. L. Lebowitz and E. H. Lieb: Striped phases in two dimensional dipole systems, Physical Review B 76, 184426 (2007).

[19] M. Grousson, G. Tarjus, and P. Viot: Phase diagram of an Ising model with long-range frustrating interactions: A theoretical analysis Physical Review E 62, 7781 (2000).

[20] P. C. Hohenberg and J. B. Swift: Metastability in fluctuation-driven first-order transitions: Nucleation of lamellar phases, Phys. Rev. E 52, 1828 (1995).

[21] E. H. Lieb and M. Loss: Analysis. Second Edition (American Mathematical Society, 2001).

[22] J. L. Lebowitz and O. Penrose: Rigorous Treatment of the Van Der Waals-Maxwell Theory of the Liquid-Vapor Transition, Journal of Mathematical Physics 7, 98-113 (1966).

[23] L. Leibler: Theory of Microphase Separation in Block Copolymers, Macromolecules 13, 1602 (1980).

[24] A. B. MacIsaac, J. P. Whitehead, M. C. Robinson, and K. De'Bell: Striped phases in twodimensional dipolar ferromagnets Physical Review B 51, 16033 (1995).

[25] W. L. McMillian: Landau theory of charge-density waves in transition-metal dichalcogenides Physical Review B 12, 1187 (1975). 
[26] C. B. Muratov: Theory of domain patterns in systems with long-range interactions of Coulomb type, Physical Review E 66, 066108 (2002).

[27] T. Ohta and K. Kawasaki: Equilibrium morphology of block polymer melts, Macromolecules 19, 2621-2632 (1986).

[28] M. Seul and D. Andelman: Domain Shapes and Patterns: The Phenomenology of Modulated Phases, Science 267, 476 (1995).

[29] B. Spivak and S. A. Kivelson: Phases intermediate between a two-dimensional electron liquid and Wigner crystal, Phys. Rev. B 70, 155114 (2004).

[30] B. Spivak and S. A. Kivelson: Transport in two dimensional electronic micro-emulsions, Ann. Phys. (N.Y.) 321, 2071 (2006).

[31] A. D. Stoycheva and S. J. Singer, Physical Review Letters 84, 4657 (2000). 